\title{
DEMONSTRATION OF FIELD EMISSION CATHODE OPERATION IN A PLASMA ENVIRONMENT
}

\author{
Christopher A. Deline ${ }^{*}$, Thomas M. Liu, David P. Morris ${ }^{*}$, and Brian E. Gilchrist ${ }^{\dagger}$ \\ University of Michigan, Ann Arbor, Michigan, 48109
}

\begin{abstract}
Field emission cathodes are being considered for use on low-power electrostatic and electromagnetic propulsion systems as well as on electrodynamic tether systems. In these systems, field emission cathodes prevent negative spacecraft charging by emitting a lowpower electron beam that is accommodated by the ambient plasma. Prototype cathodes were tested at the University of Michigan as part of a Student Space Systems Fabrication Laboratory satellite project. This paper presents experimental emission performance results of molybdenum and carbon nanotube field emission cathodes operating in a krypton plasma of density $n_{e}=1 \times 10^{11} \mathrm{~m}^{-3}$ and temperature $T_{e}=0.5-1.0 \mathrm{eV}$.
\end{abstract}

\section{Introduction/ Background}

CIELD emission cathodes (FEC) are a group of vacuum electronic devices designed to generate an electron beam P through quantum mechanical tunneling, as compared with conventional thermionic emission. These devices provide significant power and mass savings over conventional electron emission systems for low-current (microamp to milli-amp) applications. Such characteristics make FECs attractive for many proposed electric space propulsion systems ${ }^{1}$. Several technologies of FECs would be compatible with in-space electric propulsion techniques such as electrodynamic tethers and electrostatic thrusters, with the two examples considered in this paper being molybdenum "Spindt-type" cathodes ${ }^{2}$ and carbon nanotube (CNT) cathodes ${ }^{3}$. A sample cathode of each type was obtained by the Student Space Systems Fabrication Laboratory (S3FL) at the University of Michigan (UM) as part of a student-led Space Shuttle Get Away Special experiment ${ }^{4}$, and these cathodes were operated in a lowdensity plasma environment.

Both device technologies tested by the S3FL group have each undergone considerable ground testing and characterization in a variety of environments by other groups ${ }^{5,6}$. The Spindt cathode is a well-documented device fabricated by SRI International and consists of micron-diameter emitting cones etched into a molybdenum substrate with a surrounding gate electrode. The small gate-tip separation allows high current densities of approximately 1-2 $\mathrm{A} / \mathrm{cm}^{2}$ for gate-tip bias levels less than $100 \mathrm{~V}$, making these cathodes highly efficient in terms of emission current per applied voltage. Recent advances in coated silicon construction have led to devices that have successfully been qualified for spaceflight aboard the ESA's Rosetta mission ${ }^{7}$ and have successfully undergone lifetime testing for 6000 hours in consideration for ESA's upcoming SMART-2 mission. ${ }^{8,9}$ The Spindt cathodes aboard Rosetta are integrated into a mass spectrometer instrument and are not exposed to an external space environment. However, the proposed usage aboard SMART-2 would be for micropropulsion neutralization.

Carbon nanotube cathodes have also undergone rigorous ground testing as shown in a recent lifetime test ${ }^{6}$ led by Busek Co. In this test, a number of CNT cathodes withstood a battery of tests including 13,000 hours of continuous operation, exposure to $1 \times 10^{-4}$ Torr oxygen, random vibration, and UV exposure. While a CNT cathode does require a turn-on voltage around five times greater than a Spindt cathode, comparable emission current has been achieved

\footnotetext{
* Graduate Student, AIAA Student Member

${ }^{\dagger}$ Professor, Electrical Engineering and Space Systems, AIAA Associate Fellow
} 
from the same device form factor. CNT cathodes are also considered for use aboard several space missions including the LISA precursor mission SMART- $2^{10}$.

To the authors' knowledge, no test has previously been conducted using either of these device technologies in ambient plasma similar in density to the Earth's ionosphere. The purpose of the study presented in this paper is to characterize the performance of these FEC technologies to determine their feasibility for low Earth orbit (LEO) applications. In the study, the two FECs were exposed to a krypton plasma of density $1 \times 10^{11} \mathrm{~m}^{-3}$ and electron temperature about $0.5-1.0 \mathrm{eV}$. The plasma was produced by a Busek hollow cathode assembly that was held at a distance of $5 \mathrm{~m}$ from the FECs. Current-voltage characterization curves were taken with both FECs with the plasma source both on and off. Results and analysis of these tests are discussed below.

\section{Experiment Setup}

\section{A. Devices Tested}

The SRI Inc. Spindt cathode shown in Figure 1 has a standard 10-mm diameter form factor that conforms to a TO5 transistor standard. This device contains an array of 50,000 molybdenum tips co-aligned with holes in a molybdenum grid. Such alignment allows for very low leakage current to the gate. Very small gate-tip separations (a few microns) are possible, which reduce the required operating voltage. The Busek Co. CNT cathode was developed to conform to the same 10-mm diameter form factor of the Spindt cathode, thereby allowing easier integration of both types of devices into a single experiment. Each CNT cathode consists of a randomly distributed mat of multi-wall carbon nanotubes deposited onto a conducting substrate. A metal grid of $70 \%$ porosity is suspended 100 microns above the CNT layer, with electron current extracted by applying a high voltage $(400-800 \mathrm{~V})$ from the base to the grid. The small basegrid gap along with the CNT sharpness magnifies the electric field strength. Approximately $30 \%$ of the current is collected by the metal extraction grid. The Busek CNT cathode is also shown in Figure 1.

The two cathodes were housed inside a Small Vacuum Protective Enclosure (SVPE) vacuum vessel, shown in Figure 2. Developed by S3FL for FEGI (Field Emission Get-Away-Special Investigation), the planned G-187 Get Away Special payload, ${ }^{4}$ the SVPE hermetically seals the cathodes under vacuum (nominally at $10^{-3}$ Torr) to protect them from contamination prior to testing. The SVPE door is opened with a motor-driven, gearbox-coupled, ballscrew driveline. While opening, the fail-safe brake is disengaged, and the motor is activated. As the ballscrew turns, the stainless steel SVPE door lifts off and breaks the o-ring seal. After breaking the seal, the turning ball screw swings the door open, thus exposing the cathodes inside the SVPE to the test environment.

\section{B. Vacuum Chamber Facility}

The Small Vacuum Protective Enclosure was placed in the Large Vacuum Test Facility (LVTF) at UM's Plasmadynamics and Electric Propulsion Laboratory (PEPL) as shown in Figure 3. At $9 \mathrm{~m}$ long and $6 \mathrm{~m}$ in diameter, the LVTF employs seven CVI TM-1200 nude cryopumps with a combined pumping speed of 500,000 l/s for air. Peak vacuum levels of $10^{-7}$ Torr were achieved during this test, with an average background pressure of $1 \times 10^{-6}$ Torr. The SVPE was mounted at the rear of the vacuum chamber at a $5-\mathrm{m}$ separation from the hollow cathode plasma source. Electrical wiring for both cathode bias lines and for the SVPE door motor was routed through a vacuum feedthrough. 

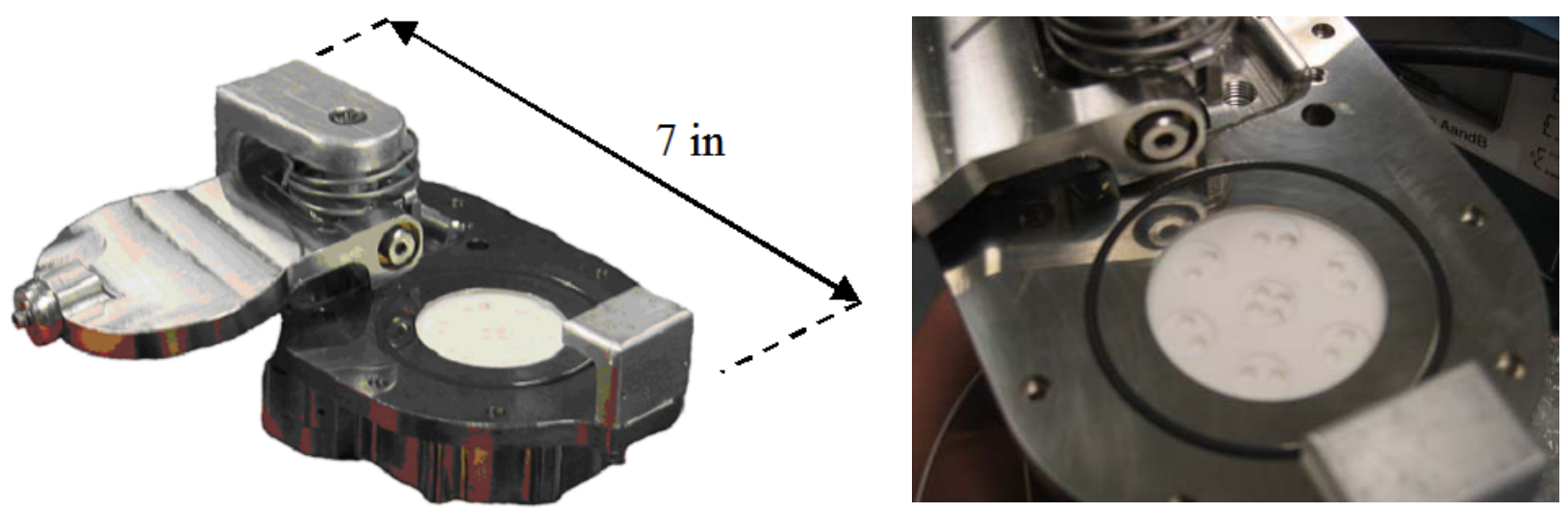

Figure 2: Small Vacuum Protective Enclosure (left) and view of interior mounting interface for FECs on TO5 headers (right).

Ambient plasma in the LVTF chamber was provided by two hollow cathode devices shown in Figure 4: one produced by Busek and the other by Moscow Aviation Institute (MAI). The two hollow cathodes were present for redundancy, with only the Busek hollow cathode being operated at the time of this test. The operating conditions of the hollow cathode during this test included a krypton flow rate of $16-20 \mathrm{sccm}(1.3 \mathrm{mg} / \mathrm{s})$ and a keeper bias of $42 \mathrm{~V}$ at $0.5 \mathrm{~A}$. Langmuir probe diagnostics in the hollow cathode far-field suggested that plasma conditions near the SVPE had a krypton ion density about $1 \times 10^{11} \mathrm{~m}^{-3}$, electron temperature about $0.5-1.0 \mathrm{eV}$, and plasma potential of $2.2 \mathrm{~V}$. These conditions were consistent with prior experiments ${ }^{11}$ and closely approximated the density of the Earth's ionosphere, which ranges from $1 \times 10^{10}-1 \times 10^{13} \mathrm{~m}^{-3}$. A separate experiment was conducted in the vacuum chamber concurrently with this test, but the two experiments were separated by a distance of 500-1000 Debye lengths and did not interfere with one other.
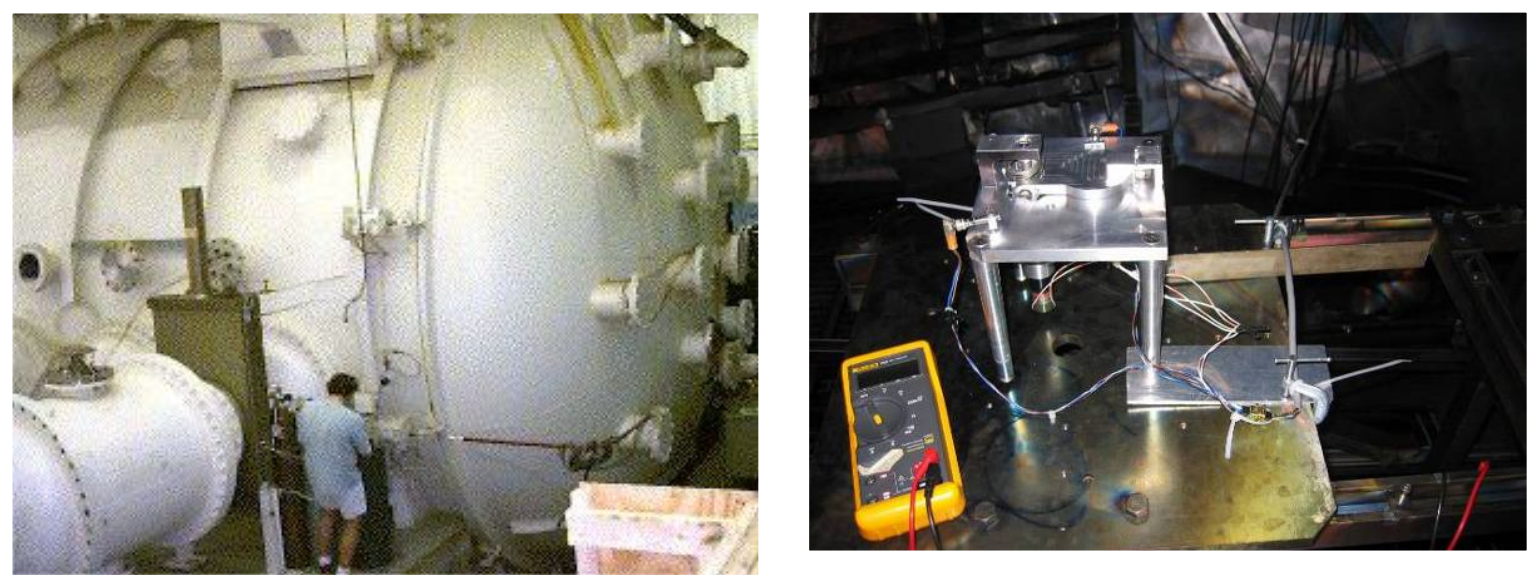

Figure 3: Facilities and equipment used for field emission testing. The Large Vacuum Test Facility at PEPL (left). Small Vacuum Protective Enclosure (right).

\section{Experiment Configuration}

The electrical configuration of the FECs is shown in Figure 5 and includes grounded grids and a negative bias on the emission surface with respect to chamber ground. Current and voltage measurements were acquired with three Keithley 2000 digital multimeters (DMM), which monitored cathode current, grid current, and grid bias. The plasma was floating at the natural plasma potential based on the density and electron temperature. The two FECs in this test were operated sequentially, not in tandem. Gate-tip bias $\mathrm{V}_{\text {grid }}$ was provided by a high voltage (500 V) DCDC converter, which was automated by a prototype digital-to-analog converter and microprocessor board. Note that the grid-tip bias was monitored in parallel by a Keithley 2000 DMM, while the prototype equipment was being 
developed for flight as part of the FEGI experiment. Data acquisition at $0.5 \mathrm{~Hz}$ was accommodated by LabVIEW software and a GPIB connection with the Keithley 2000 DMMs.

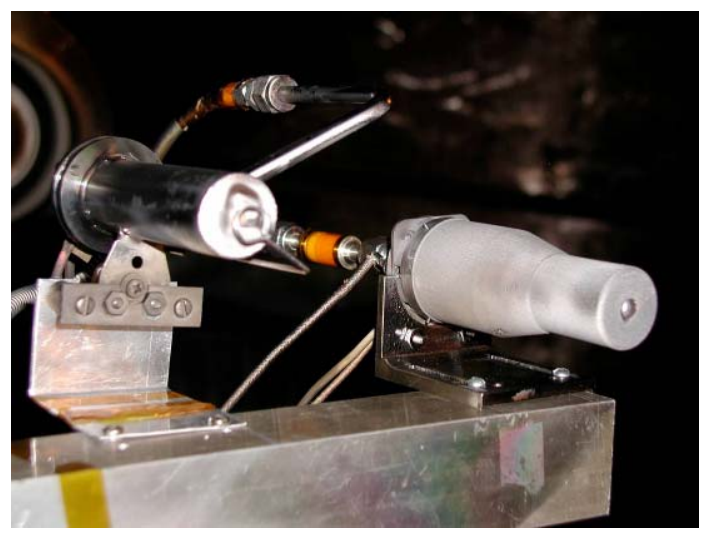

Figure 4: MAI hollow cathode (left) and Busek hollow cathode (right). The two hollow cathodes provided an ambient plasma environment of ion density $\sim 1 \times 10^{13} \mathrm{~m}^{-3}$ in the hollow cathode near field and $\sim 1 \times 10^{11} \mathrm{~m}^{-3}$ ion density in the far-field.

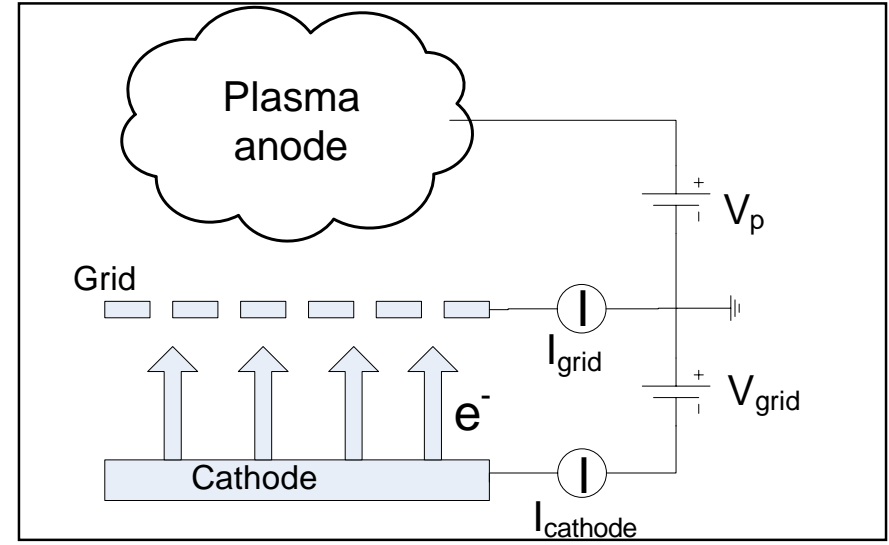

Figure 5: Experiment setup. The FEC shown here is emitting into an ambient plasma. No anode was present in the LVTF during vacuum operation. Electrons were collected by surrounding grounded metal components.

No fixed anode was present during these tests. During the plasma immersion test, ambient plasma provided by the krypton-fed hollow cathode served as the anode. In this configuration, a plasma potential $\mathrm{V}_{\mathrm{p}}=2.2 \mathrm{~V}$ was set up between ambient plasma and the grounded grid due to the potential drop within the sheath. This potential accelerated electrons past the cathode's grid into the surrounding plasma, where they were accommodated by the plasma. Analytical calculations showed that current levels were low enough that the plasma would have been able to accommodate this charge without space-charge limit reflection. The measured currents to the gate $\left(\mathrm{I}_{\text {grid }}\right)$ and cathode ( $\mathrm{I}_{\text {cathode }}$ ) supported this assumption, although no direct measurement of plasma anode current was possible in this experimental configuration. The FEC's emission current into the ambient plasma was calculated to be the difference between the current to the cathode and the current collected at the grid.

\section{Analysis \& Discussion of Results}

The cathode tests were conducted as a target-of-opportunity test in conjunction with a separate primary investigation into charged conductors immersed in flowing plasma. As such, the cathode tests were not the primary focus during the testing campaign. Also, additional goals of the cathode tests were to include prototype hardware developed by students and to maximize the amount of student involvement in the experiment. This philosophy led to several non-optimal test results: 1.) The cathodes were not operated at maximum current output and did not achieve the theoretical maximum current density during operation. 2.) A baseline vacuum operation of the molybdenum Spindt cathode was not obtained, only data from operation in plasma. In order to compare with theory, the Spindt experiment data is merely compared with manufacturer-provided characterization curves for several representative devices. 3.) The lifetime of both cathodes was limited due to current degradation (CNT) and persistent short-circuits (Spindt) that developed several hours into the test. This occurrence was due in part to handling procedures used during the test as well as the impure nature of the test environment. The results presented here are not lifetime results but characterization curves over a short time period in plasma and in vacuum.

\section{CNT Cathode Results}

After the LVTF chamber was pumped down to its final pressure of $1 \times 10^{-6}$ Torr, the motorized door of the Small Vacuum Protective Enclosure was opened to expose the FECs to vacuum. Baseline CNT cathode operation in vacuum was initiated following an outgassing period of an hour. During baseline vacuum measurement, a $300 \mathrm{~V}-$ $500 \mathrm{~V}$ voltage ramp profile was applied to the CNT cathode followed by a 10 -minute, constant $500-\mathrm{V}$ soak. The hollow cathode plasma source was then turned on, immersing the CNT cathode in a low-density plasma. A similar $300 \mathrm{~V}-500 \mathrm{~V}$ voltage ramp was used to characterize the CNT cathode in plasma, followed by a 10-minute, constant $500-\mathrm{V}$ soak. 
The current-voltage profile obtained with both vacuum and plasma conditions is compared with the following equation for Fowler-Nordheim current ${ }^{12}$ in Figures 6 and 7:

$$
I_{F N}=\frac{1.5 \times 10^{-6}}{\phi}\left(\frac{\beta V}{D}\right)^{2} \exp \left(\frac{10.4}{\sqrt{\phi}}\right) \exp \left(\frac{-6.44 \times 10^{9} \phi^{1.5}}{\beta V / D}\right) * A
$$

where $\Phi$ is the work function of the emitting material, $\beta$ is the geometric enhancement factor for surface geometry, $V$ is the bias voltage, $D$ is the gap distance, and $A$ is the emission surface area. In addition to the Fowler-Nordheim current, an additional $I=V / R_{\text {shunt }}$ term was included to account for the cathode-grid shunt resistance of the device and experiment setup.

Table 1 below lists the Fowler-Nordheim parameters used to arrive at the analytical curve fits to the CNT cathode experimental data. Both sets of parameters compare favorably with experimental reference cases taken from the literature. These reference values are also given in Table 1.

Table 1: Parameters for Fowler-Nordheim equation found in the literature (theory) and used to fit curves to CNT cathode data (experiment).

\begin{tabular}{lccccc}
\hline Device & $\Phi$ & $\beta$ & $D$ & Area & $R_{\text {shunt }}$ \\
\hline $\begin{array}{l}\text { Carbon nanotube } \\
\text { (reference) }\end{array}$ & 5.1 & $110+/-20$ & $1 \times 10^{-4} \mathrm{~m}$ & $3 \times 10^{-15} \mathrm{~m}^{2}$ & N/A \\
$\begin{array}{l}\text { Carbon nanotube } \\
\text { (exp. in vacuum) }\end{array}$ & 5.1 & 104 & $1 \times 10^{-5} \mathrm{~m}$ & $8 \times 10^{-15} \mathrm{~m}^{2}$ & $1.9 \mathrm{G} \Omega$ \\
$\begin{array}{l}\text { Carbon nanotube } \\
\text { (exp. in plasma) }\end{array}$ & 5.1 & 115 & $1 \times 10^{-5} \mathrm{~m}$ & $2 \times 10^{-15} \mathrm{~m}^{2}$ & $1.2 \mathrm{G} \Omega$ \\
\hline
\end{tabular}

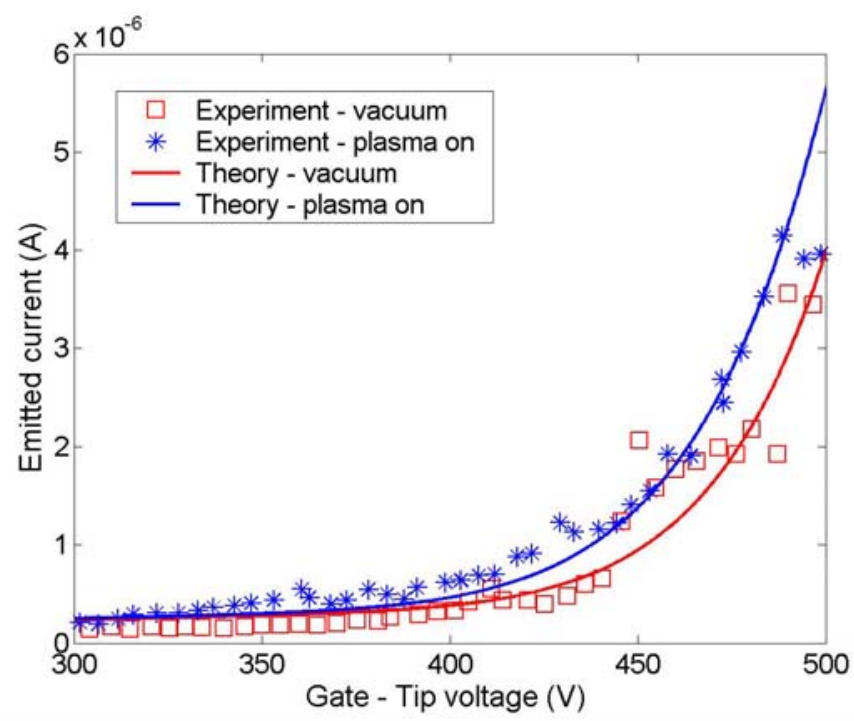

Figure 6: Current - Voltage plot for a CNT cathode. Shows operation in vacuum and in plasma, compared with standard Fowler-Nordheim theory.

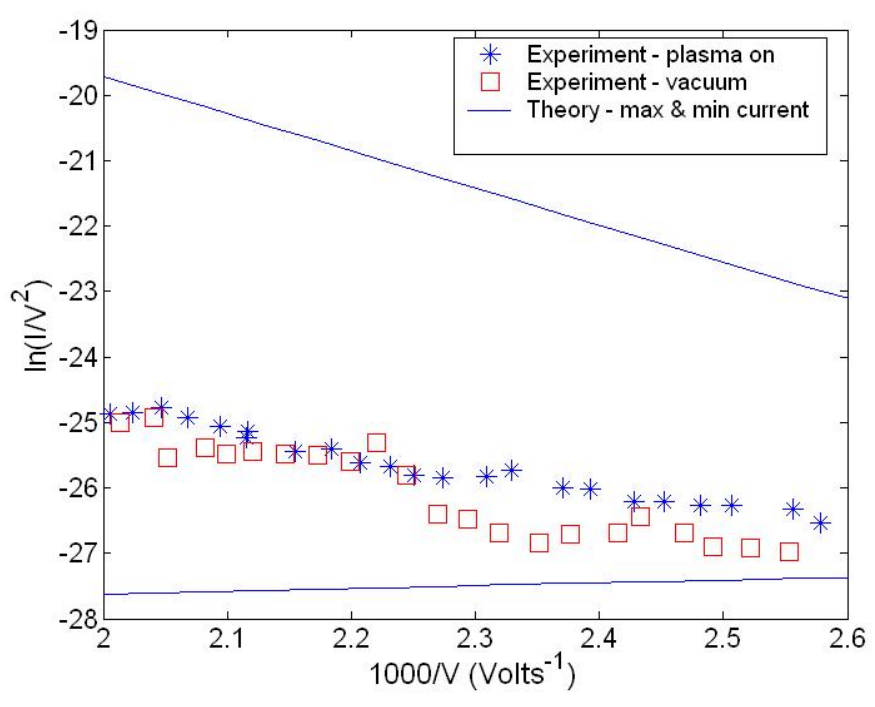

Figure 7: Fowler - Nordheim plot with CNT experiment data. Also shown is theoretical minimum and maximum current for parameters taken from Table 1: CNT theory. 
As Figure 6 shows, the CNT cathode operating in vacuum and operating in plasma shows similar results, both of which are shown graphically in Figure 7 to be within the bounds of prior reference experiments. This boundary of prior reference experiments is derived from the minimum current response and maximum current response of a theoretical CNT cathode operating within the range of reference parameters given in Table 1. The immersion of a CNT cathode into a neutral krypton plasma in the density range of the Earth's ionosphere thus did not have significant impact on the current-voltage character of the CNT cathode for the short duration of this characterization test.

Given a longer period of time over the course of several I-V sweeps, the CNT cathode did degrade under both vacuum and plasma conditions. This trend has also been recorded by Gasdaska et al. ${ }^{6}$ and was attributed to either removal of CNTs from the substrate due to the electric field force on the CNTs or localized overheating of the CNTsubstrate interface. Since our experiment was not optimized to provide a long duration lifetime test, the source of this degradation effect was not investigated any further.

\section{E. Spindt Cathode Results}

Once operations were completed with the CNT cathode, the Spindt cathode was conditioned and operated in vacuum. A current-voltage characterization sweep in vacuum was not possible due to a persistent gate-tip short that was not cleared until the plasma source was turned on. Once stable emission was achieved in the $1 \times 10^{11} \mathrm{~m}^{-3}$ density plasma, a currentvoltage characterization sweep was obtained, which is shown as a Fowler-Nordheim plot in Figure 8. This data is shown along with a range of manufacturer-provided characterization sweeps for Spindt cathodes operating in vacuum. While a direct comparison between vacuum operation and plasma operation of the same cathode is not possible, the results for the Spindt cathode operating in plasma lie within the range of expected results for a standard Spindt cathode operating in vacuum. It can thus be concluded that the presence of plasma did not affect the characteristics of electron emission more significantly than device-to-device manufacturing variation. Table 2 shows Fowler-Nordheim parameters for typical Spindt cathodes operating in vacuum, along with Fowler-Nordheim parameters used to provide the linear fit shown in Figure 8.

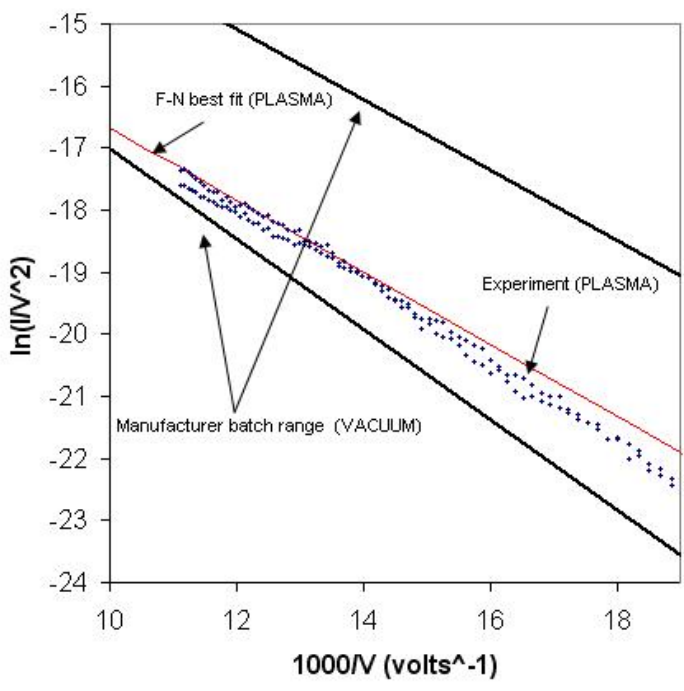

Figure 8: Spindt device experimental data taken in a low-density plasma environment. Compared to upper and lower boundaries of manufacturer-provided vacuum calibration data. Linear fit shows $F-N$ theory given experimental parameters in Table 2.

Table 2: Parameters for Fowler-Nordheim equation found in the literature (theory) and used to fit curves to Spindt cathode data (experiment).

\begin{tabular}{lccccc}
\hline Device & $\Phi$ & $\beta$ & $D$ & Area & $R_{\text {shunt }}$ \\
\hline $\begin{array}{l}\text { Spindt-type } \\
\text { (reference) }\end{array}$ & $4.5-4.9$ & $100-200$ & $1 \times 10^{-6} \mathrm{~m}$ & $4 \times 10^{-16} \mathrm{~m}^{2}$ & N/A \\
$\begin{array}{l}\text { Spindt-type (exp. in } \\
\text { plasma) }\end{array}$ & 4.9 & 120 & $1 \times 10^{-6} \mathrm{~m}$ & $4 \times 10^{-17} \mathrm{~m}^{2}$ & $2.0 \mathrm{G} \Omega$ \\
\hline
\end{tabular}

During operation, a peak emission current of $0.375 \mathrm{~mA}$ was recorded at $90 \mathrm{~V}$, which is consistent with prior experiments on Spindt cathodes in vacuum. All experimentally determined Fowler-Nordheim parameters follow closely to the reference case, with the possible exception of the estimated emission surface area, which was measured to be an order of magnitude lower than the maximum possible area presented in the reference case. 


\section{Conclusions and Further Study}

In the tests considered in this paper, two technologies of field emission cathodes were exposed to neutral plasma similar in density to Earth's ionosphere. The emission characteristics of both cathodes did not differ significantly from vacuum operation, and both fit within Fowler-Nordheim theory given reasonable equation parameters. While lifetime effects were not studied in this test, operation of both cathodes for a period of several hours was demonstrated, and several secondary objectives such as testing prototype electronics and student involvement were also accomplished.

\section{Acknowledgments}

Special thanks go to all of the corporate and government sponsors of the Student Space Systems Fabrication Laboratory, including Lockheed Martin, the Air Force Academy, Busek Inc., NASA Marshall Space Flight Center, the University of Michigan (UM), Pennsylvania State University (PSU), and the Air Force Research Laboratory (Hanscom AFB). Thanks also go to the many student volunteers that have assisted in the design and construction of the FEGI experiment both at UM and PSU. This work was supported by the U.S. Air Force's University Nanosat program. 


\section{References}

${ }^{1}$ Aguero, V.M. and Adamo, R.C., “Space Applications of Spindt Cathode Field Emission Arrays” Spacecraft Charging Technology Conference, Air Force Research Lab AFRL-VS-TR-20001578, 2000, pp. 347-352

${ }^{2}$ Spindt, C.A., “A thin-film field-emission cathode”, Journal of Applied Physics, 39, (7), 3504, June 1968

${ }^{3}$ Gamero-Castaño, M. et al., "Electron field emission from carbon nanotubes and its relevance in space applications,” AIAA Joint Propulsion Conference 2000-3263 AIAA, 2000

${ }^{4}$ Deline, C. et al., "Field Emission Cathodes Used in the FEGI Get Away Special Shuttle Mission”, AIAA Joint Propulsion Conference, 2004

${ }^{5}$ Marrese, C. et al., "In-FEEP Thruster Ion Beam Neutralization with Thermionic and Field Emission Cathodes" IEPC-01-290 (2001)

${ }^{6}$ Gasdaska, C. et al., “Testing of Carbon Nanotube Field Emission Cathodes”, AIAA Joint Propulsion Conference 2004-3427 AIAA, 2004

${ }^{7}$ Kent, B. J., Huq, Dominey and Morse, “The use of microfabricated field emitter arrays in a high precision mass spectrometer for the Rosetta mission” 3rd Round Table on Micro/Nano Technologies for Space European Space Technology Centre, Noordwijk, Netherlands, 2000

${ }^{8}$ Aplin K. L., Collingwood and Kent, "Reliability tests of gated silicon field emitters for use in space"

J. Phys. D: Appl. Phys. 37 2009-17 (2004)

${ }^{9}$ Kent, B. J. et al., “A field effect spacecraft neutralizer for the LISA Pathfinder mission” Class. Quantum Grav. 22 (2005) S483-S486

${ }^{10}$ Hruby, V. et. al, “Colloid Thrusters for the New Millennium ST7 DRS Mission,” IEEEAC Conference, Big Sky, MT, Paper \#1329, 2004

${ }^{11}$ Bilen, S.G., Domonkos, M.T., and Gallimore, A.D., “The Far-Field Plasma Environment of a Hollow Cathode Assembly”, AIAA Joint Propulsion Conference 1999-2861, AIAA, Washington D.C., 1999

${ }^{12}$ Fowler, R. H., and L. Nordheim, Proc. R. Sot. London Ser. A 119, 173 (1928); L. Nordheim, ibid. 121, 626 (1928)

${ }^{13}$ Bonard, J., and Klinke, “Degradation and failure of carbon nanotube field emitters,” Physical Review B vol. 67 number 115406, 2003

${ }^{14}$ Utsumi, T., “Vacuum Microelectronics - What’s new and exciting” IEEE trans. elec. dev. 38 (10), 1991 